\title{
July 2014 Pulmonary Case of the Month: Where Did It Come From?
}

\author{
Colin B. Fitterer, MD \\ James M. Parish, MD \\ Mayo Clinic Arizona \\ Scottsdale, AZ
}

\section{History of Present IIIness}

A 67 year old man presented with worsening cough and shortness of breath. He has a history of metastatic colon cancer first diagnosed in 2010. He was treated with radiation and chemotherapy (FOLFOX) but unfortunately developed new pulmonary nodules in October, 2013 which were metastatic colon cancer on biopsy. In February 2014 he developed a right parietal brain mass which was resected. Thoracic CT scan at that time showed progression of the pulmonary nodules. He has also noted a 30 pound weight loss over the past 6 months and an enlarging right supraclavicular lymph node.

\section{$P M H, F H, S H$}

In addition to the colon cancer, he has previous diagnoses of type 2 diabetes mellitus, hypertension, allergic rhinitis, and vitamin $D$ deficiency. He is married and a recently retired railroad engineer. He has no history of tobacco use. There is a positive family history of lung cancer but no colon cancer.

\section{Physical Examination}

Vital Signs: Temperature 36.8, pulse 98, respirations 18, blood pressure 144/70, SpO2 $91 \%$ on $3 \mathrm{~L}$ via nasal cannula.

Pertinent findings include:

A large firm and fixed right supraclavicular lymph node that is nonpainful on palpation.

Diminished breath sounds across all right posterior lung fields with dullness to percussion.

Palpable liver edge is palpable approximately $2 \mathrm{~cm}$ below the right costal margin.

\section{Laboratory Analysis}

Admission laboratory values include a hemoglobin of $11.1 \mathrm{~g} / \mathrm{dL}$ but with a normal white blood cell count and platelet count. Electrolytes, blood urea nitrogen, creatinine, and liver enzymes were all within normal limits. Serum chemistries are within normal limits.

\section{Radiography}

A chest x-ray (Figure 1A) and chest CT (Figure 1B) were performed. 


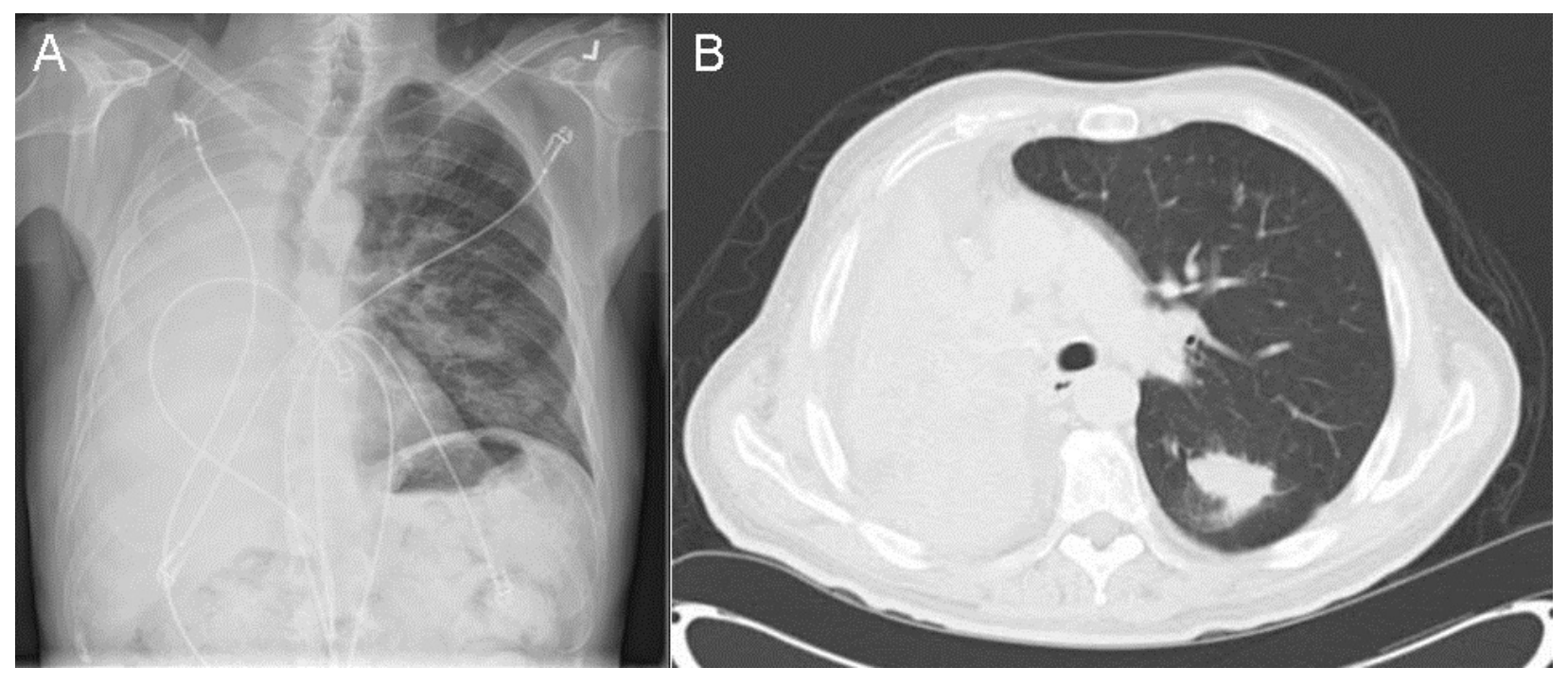

Figure 1. Admission AP (Panel A) and representative image from the thoracic CT scan (Panel B).

Which of the following is the best interpretation of the radiographic findings?
1. Large right pleural effusion
2. Right lung atelectasis
3. Right lung pneumonia
4. Right lung pulmonary edema
5. None of the above 


\section{Correct! \\ 2. Right lung atelectasis}

The chest x-ray shows evidence of volume loss on the right (Figure 2).

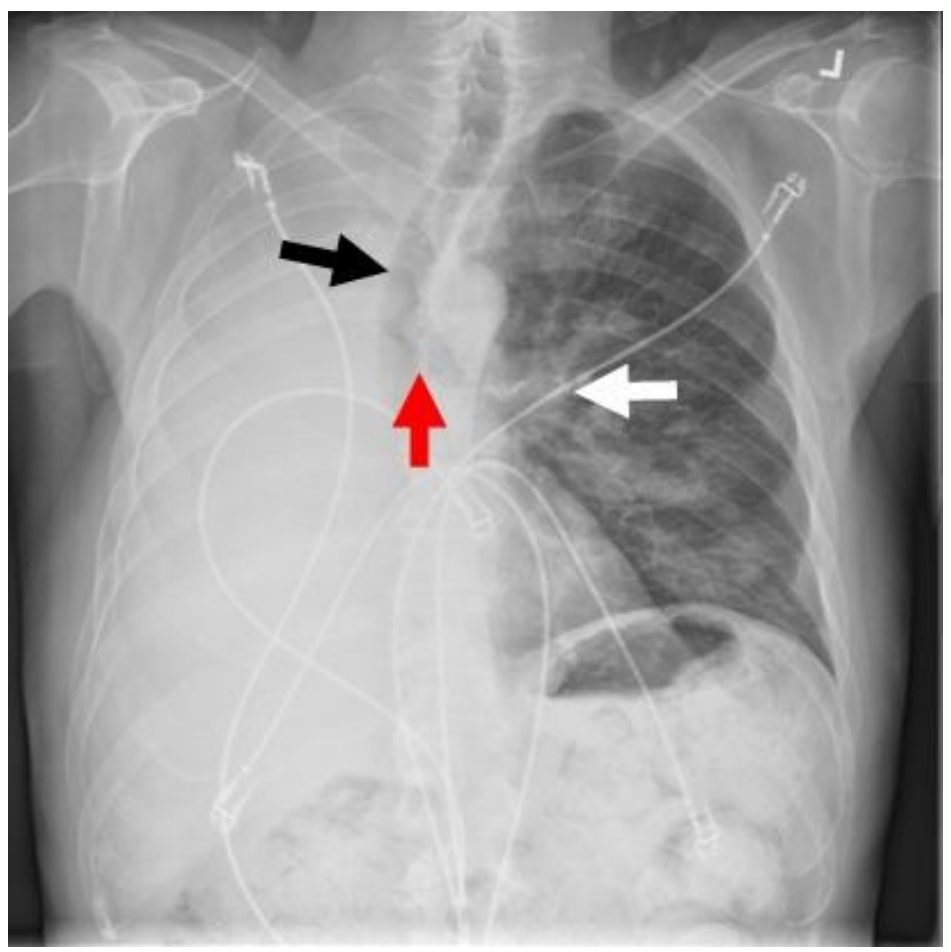

Figure 2. Admission AP of chest showing shift of the trachea to the right (black arrow), shift of the carina to the right (red arrow) and shift of the left hilum to the right (white arrow).

Atelectasis is associated with volume loss while pleural effusion, pneumonia and pulmonary edema are not.

What is the next step in evaluating the patient?

1. Bronchoscopy

2. Left lung mass biopsy

3. Liver biopsy

4. Right lung needle biopsy

5. Supraclavicular lymph node biopsy 


\section{Correct! \\ 1. Bronchoscopy}

The patient has widely metastatic colon cancer. Bronchoscopy is indicated to evaluate the cause of the central bronchial obstruction resulting in the right lung atelectasis. Biopsy of the cervical lymph node, liver or the left lung mass seen on CT scan will likely show metastatic colon cancer. Bronchoscopy would be diagnostic and possibly therapeutic (1).

Which of the following are potential causes of central airway obstruction in this patient?

1. Endobronchial metastasis

2. Lymphadenopathy

3. Mucus plugging

4. Primary lung cancer

5. All of the above 


\section{Correct!}

\section{All of the above}

Each of these causes is possible (1). Patients with cancer are known to be more likely to develop a second cancer. Lymphadenopathy resulting in extrinsic compression of the bronchus or mucus plugging are also possible. Bronchoscopy revealed a large mass obstructing the right mainstem bronchus (Figure 3).

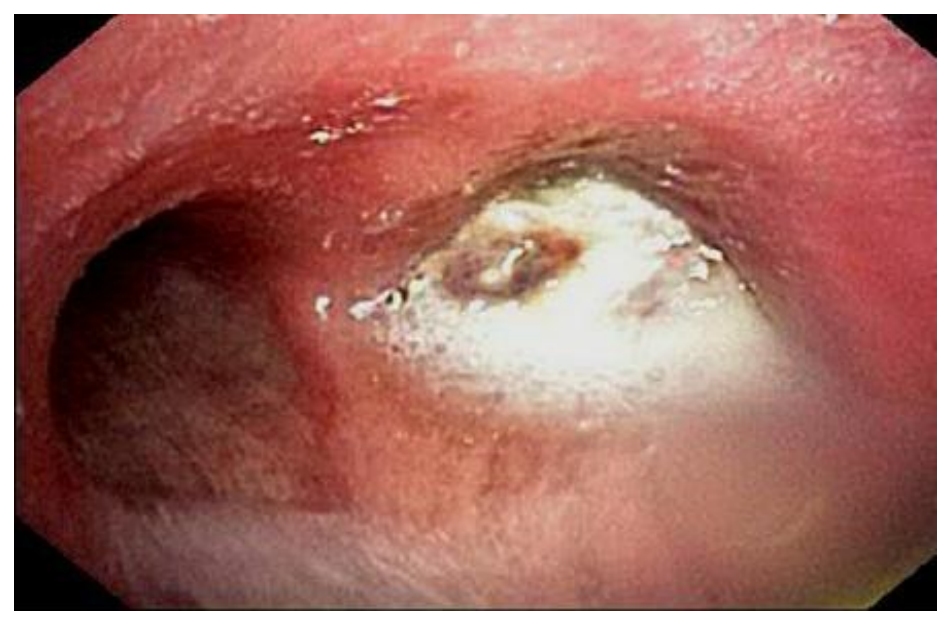

Figure 3. View from the trachea showing right mainstem bronchial obstruction.

Which of the following is indicated?

1. Biopsy the mass

2. Laser the mass

3. Mechanically debulk the mass

4. Suction secretions

5. All of the above 


\section{Correct! \\ 5. All of the above}

Biopsy of the obstruction was consistent with metastatic colon cancer (Figure 4).

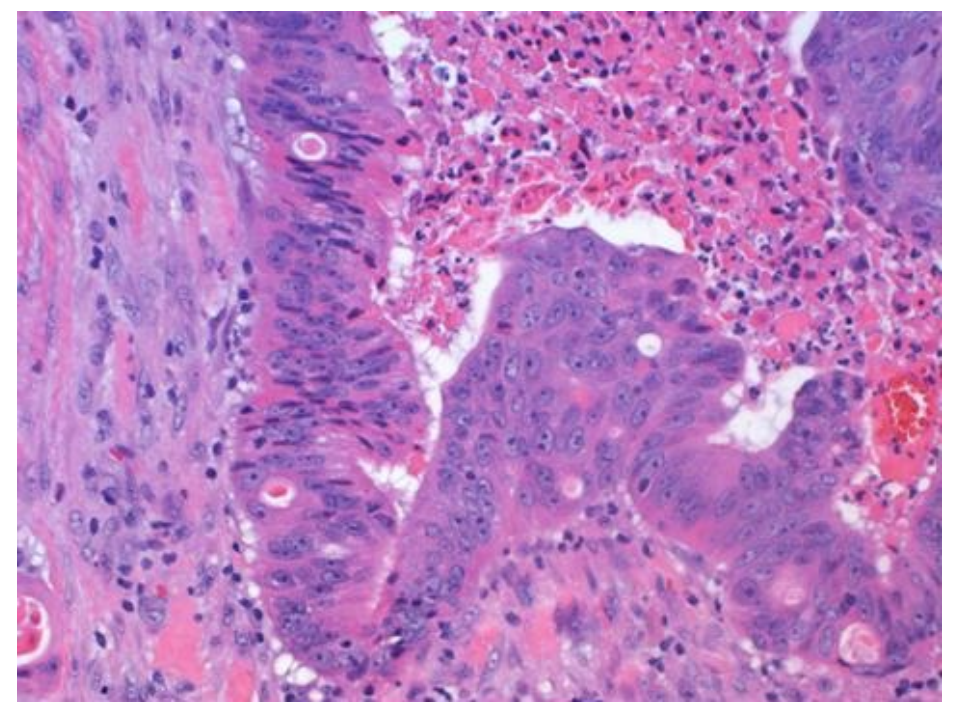

Figure 4. Histology of the bronchial biopsy consistent with metastatic colon cancer.

Our patient had significant improvement in his dyspnea from debulking of the soft, necrotic tumor in right mainstem bronchus with argon plasma coagulation to control bleeding and extensive suctioning of purulent airway secretions. His chest x-ray also showed significant improvement (Figure 5).

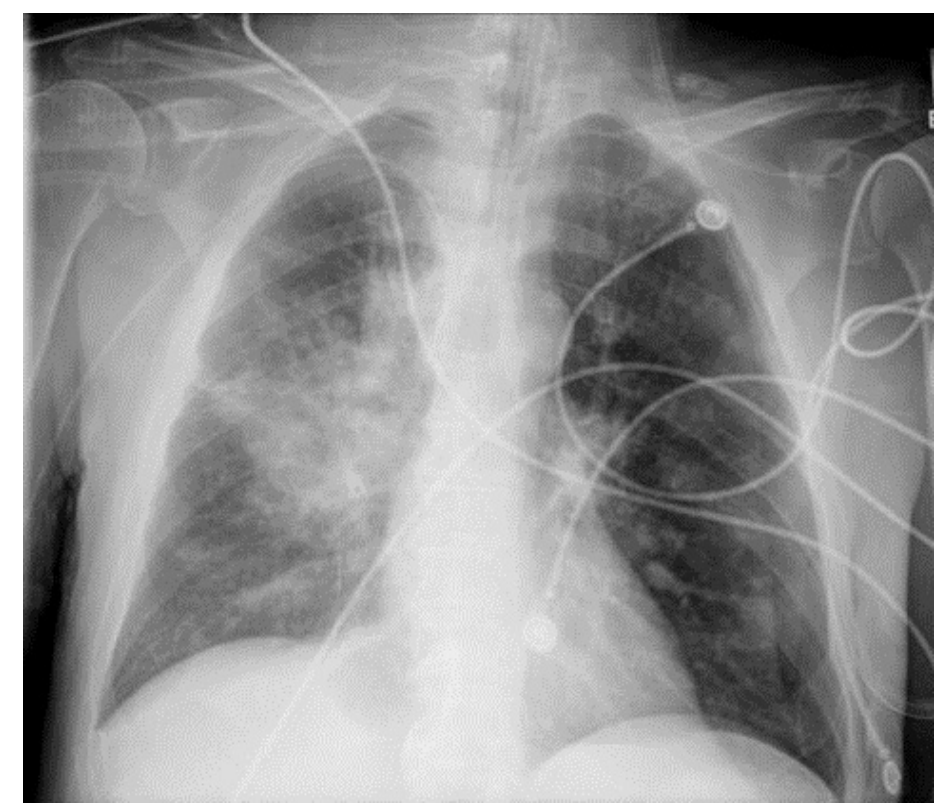

Figure 5. Chest x-ray taken after tumor debulking. 
The prevalence of endobronchial metastases is about $2 \%$ based on autopsy studies (2). However, the prevalence increases to $18-42 \%$ in patients with pulmonary metastases. Endobronchial metastases are most frequently observed in cancers from the breast, kidney, colorectal, uterine cervix, sarcoma and skin cancers (2). In a review of the literature, the mean time from diagnosis of the primary tumor was 50 months (range 0300 months) and mean survival time from the diagnosis of endobronchial metastasis was 15.2 months (range 0-150 months) (2). Endobronchial metastases from colon cancer reflects these trends. In a series of 42 patients, average survival from primary diagnosis: 70 months (23-245) but median survival after endobronchial metastases were detected was only 14 months (3-40) (3).

The take home messages are that endobronchial metastases tend to arise in the later stages of disease and have increased incidence when pulmonary metastasis are present. Interventional bronchoscopy may offer significant palliative benefit.

\section{References}

1. Ernst A, Feller-Kopman D, Becker HD, Mehta AC. Central airway obstruction. Am J Respir Crit Care Med. 2004;169:1278-97. [CrossRef] [PubMed]

2. Sørensen JB. Endobronchial metastases from extrapulmonary solid tumors. Acta Oncol. 2004;43(1):73-9. [CrossRef] [PubMed]

3. Fournel C, Bertoletti L, Nguyen B, Vergnon JM. Endobronchial metastases from colorectal cancers: natural history and role of interventional bronchoscopy. Respiration. 2009;77(1):63-9. [CrossRef] [PubMed] 